\title{
Alcohol Consumption, Types of Alcoholic Beverages and Risk of Venous Thromboembolism - The Tromsø Study
}

Hansen-Krone: Alcohol consumption and VTE

Ida J. Hansen-Krone, BSc, ${ }^{*}$ Sigrid K. Brækkan, PhD, ${ }^{*}$ Kristin F. Enga, BSc, ${ }^{*}$ Tom Wilsgaard $\mathrm{PhD},{ }^{\dagger}$ and John-Bjarne Hansen $\mathrm{MD} \mathrm{PhD}^{* *}$

${ }^{*}$ Hematological research group (HERG), Department of Clinical Medicine and 'Department of Community Medicine, University of Tromsø, ${ }^{*}$ Division of Internal Medicine, University Hospital of North Norway, Tromsø, Norway.

Correspondence to: Ida Hansen-Krone, Hematological research group in Tromsø (HERG), Department of Clinical Medicine, University of Tromsø, N-9037 Tromsø, Norway. E-mail: ida.j.hansen-krone@uit.no. Telephone: +47 776 46408. Fax: +47 77644650

Word count abstract: 250

Total word count: 4970

The authors report no potential conflict of interest 


\section{Summary}

Moderate alcohol consumption has been shown to protect against cardiovascular diseases. The association between alcohol consumption, especially types of alcoholic beverages, and venous thromboembolism (VTE) is less well described. The aim of this study was to investigate the impact of alcohol consumption and different alcoholic beverages on risk of VTE. Information on alcohol consumption were collected by a self-administrated questionnaire in 26662 subjects, aged 25-97 years, who participated in the Tromsø Study, in 1994-95. Subjects were followed through September 1, 2007 with incident VTE as the primary outcome. There were 460 incident VTE-events during a median of 12.5 years of follow-up. Total alcohol consumption was not associated with risk of incident VTE. However, subjects consuming $\geq 3$ units of liquor per week had $53 \%$ increased risk of VTE compared to teetotalers in analyses adjusted for age, sex, BMI, smoking, diabetes, cancer, previous cardiovascular disease, physical activity and higher education (HR: $1.53,95 \% \mathrm{CI}$ : 1.00-2.33). Contrary, subjects with a wine intake of $\geq 3 \mathrm{u} /$ week had $22 \%$ reduced risk of VTE (HR: 0.78, 95\% CI: 0.47-1.30), further adjustment for liquor and beer intake strengthened the protective effect of wine (HR: $0.53,95 \%$ CI: 0.30-1.00). Frequent binge drinkers ( $\geq 1 /$ week) had a 17\% increased risk of VTE compared to teetotallers (HR 1.17, 95\% CI: 0.66-2.09), and a $47 \%$ increased risk compared to non-binge drinkers (HR 1.47, 95\% CI: 0.85-2.54). In conclusion, liquor consumption and binge drinking was associated with increased risk of VTE, whereas wine consumption was possibly associated with reduced risk of VTE.

Keywords: alcohol, prospective cohort, risk factors, venous thromboembolism 


\section{Introduction}

Deep vein thrombosis (DVT) and pulmonary embolism (PE) together referred to as venous thromboembolism (VTE), is a common, multifactor disease, and a serious cause of morbidity and mortality $(1,2)$. It affects $1-2$ per 1000 adults each year $(1,3)$ and is the third most common cardiovascular disease (4). Despite numerous known risk factors, still $25-50 \%$ of the events appear in the absence of predisposing factors (5). In contrast to coronary artery disease (e.g. myocardial infarction), the incidence of VTE has not declined during the last decades (1). Thus, it is vital to elucidate the impact of lifestyle factors on risk of VTE, in order modify the risk and reduce the disease burden related to VTE.

In 2004, the World Health Organization (WHO) estimated that alcohol (ethanol) was consumed regularly by about two billion people worldwide (6). Excessive alcohol intake and binge drinking are associated with major health risks and premature death. On the other hand, alcohol affects several factors involved in haemostasis, such as inhibition of platelet aggregation (7), lowering of fibrinogen (8-10), factor VII (FVII), von Willebrand factor (vWF) and plasma viscosity (10), as well as increased levels of tissue plasminogen activator (tPA) $(8,9)$, and accumulating evidence indicates that moderate alcohol consumption may have a beneficial effect on arterial cardiovascular diseases $(11,12)$.

The association between alcohol consumption and VTE is less well described, and previous studies have been somewhat inconsistent. Three prospective studies of elderly (13), young and middle-aged women $(14,15)$ and one large case-control study including both sexes $(16)$ reported an inverse association between alcohol consumption and VTE. In contrast, two other cohorts including male physicians (4) and middle-aged and elderly (17), as well as one case- 
control study of medical outpatients (18), found no association between alcohol consumption and VTE.

Beer, wine and spirits may have different effects on VTE risk. Only one of the previous studies, including elderly women only, had differentiated for type of alcoholic beverages (14). In the present study, we examined the impact of total alcohol consumption, as well as consumption of beer, wine and liquor separately, on the incidence of VTE in a large prospective cohort recruited from a general population.

\section{Materials and methods}

\section{Study population}

Participants were recruited from the fourth survey of the Tromsø Study (conducted in 199495), a single-centre prospective, population-based study, with repeated health surveys of the inhabitants of Tromsø, Norway. All inhabitants aged $>24$ years were invited, and 27158 ( $77 \%$ of the eligible population) participated. The study was approved by the regional committee for research ethics, and all participants gave written consent to participate. Subjects who did not consent to medical research $(n=201)$, subjects not officially registered inhabitants of the municipality of Troms $\varnothing$ at baseline $(n=43)$, subjects with a previous history of VTE $(n=48)$, and subjects who provided no information on any of the alcohol questions (n $=204)$ were excluded. Thus, 26662 subjects were included in the study, and followed from the date of enrolment to the end of the study period, September 1, 2007.

\section{Assessment of alcohol consumption}

Alcohol consumption habits were assessed through a self-administered questionnaire at baseline. Subjects were asked whether they were teetotalers (yes/no). If no, subjects were 
asked how many glasses of wine, beer or liquor they usually drank within a two week period. One unit corresponded to approximately 14 grams of alcohol. Total alcohol intake was categorized into teetotalers, $<1$ unit/week, 1-2 units/week, 3-6 units/week and $\geq 7$ units/week. Beer, wine and liquor consumption were categorized into teetotalers, $<1$ unit/week, 1-2 units/week and $\geq 3$ units/week. The questionnaire did not provide information on former drinking among teetotalers. A subpopulation of 21382 participants (all subjects aged $<70$ years) were also asked how often during the past 12 months they had been drinking alcohol corresponding to at least 5 small bottles of beer, a bottle of wine or a quarter bottle of liquor (binge drinking). Binge drinking was categorized into teetotalers, never (subjects who were not teetotalers and reported no binge drinking), a few times, 1-2 times per month, and once a week or more.

\section{Other measurements}

Height and weight were measured with subjects wearing light clothing and no shoes. Body mass index (BMI) was calculated as weight in kilograms, divided by the square of height in meters $\left(\mathrm{kg} / \mathrm{m}^{2}\right)$. Information on self-reported diabetes, prior cardiovascular disease (myocardial infarction, angina pectoris or stroke), current daily smoking (pipe/cigar/cigarettes), hormone therapy (self-reported current use of estrogen supplementation or oral contraceptives), higher education (education at college /university level) and physical activity ( $\geq 1$ hour per week) during leisure time was collected through a self-administered questionnaire. Information on pre-baseline cancer was obtained from the Norwegian Cancer Registry. 


\section{Venous thromboembolism ascertainment}

All first lifetime events of VTE during follow-up were identified by searching the hospital discharge diagnosis registry, the autopsy registry, and the radiology procedure registry at the University Hospital of North Norway, as previously described (19).

The medical records for each potential VTE-case were reviewed by trained personnel. For subjects derived from the hospital discharge diagnosis registry and the radiology procedure registry, an episode of VTE was verified and recorded as a validated outcome when all four of the following criteria were fulfilled: (i) objectively confirmed by diagnostic procedures (compression ultrasonography, venography, spiral-computed tomography (CT), perfusionventilation scan, pulmonary angiography or autopsy); (ii) the medical record indicated that a physician had made a diagnosis of DVT or PE; (iii) signs and symptoms consistent with DVT or PE were present; (iv) therapy with anticoagulants (heparin, warfarin, or similar agent) thrombolytics, or vascular surgery were required. For subjects derived from the autopsy registry, a VTE-event was recorded as an outcome when the autopsy record indicated VTE as a cause of death or as a significant condition.

\section{Statistical analyses}

For each participant, person-years of follow-up were accrued from the date of enrolment in the Tromsø study (1994-95), to the date a VTE-event was first diagnosed, the date the participant died or moved from the municipality of Tromsø, or to the end of the study period, September 1, 2007. Subjects who moved from the municipality of Tromsø or died during follow-up were censored from the date of death or migration. 
Statistical analysis was carried out using SPSS version 17.0 (SPSS Inc.Chicago, IL, USA).

The significance level was 0.05 . Cox proportional hazards regression models were used to estimate age- and sex-adjusted, and multivariable-adjusted, hazard ratios (HRs) for VTE by levels of total alcohol consumption, and levels of beer, wine and liquor consumption separately. The lowest consumption level was used as the reference level in each model. In the multivariable model 2, HRs were adjusted for age, sex, BMI, smoking, diabetes, cancer, history of cardiovascular diseases, educational level and physical activity. In model 3, categories of beer, wine and liquor were added to model 2 , resulting in a model where the types of alcoholic beverages were adjusted for one another in addition to all the covariates from model 2. The proportional hazard assumption was verified by evaluating the parallelism between the curves of the log-log survivor function for categories of alcohol consumption.

\section{Results}

There were 460 validated incident VTE-events during 288730 person-years of follow-up, and the overall crude incidence rate was 1.60 per 1000 person-years (95\% CI: $1.46-1.75)$. Characteristics of the patients at the time of the VTE event are shown in Table 1.

Baseline characteristic across levels of weekly alcohol intake is shown in Table 2. The teetotalers were older and had a higher proportion of concomitant diseases (diabetes, cancer and prior CVD). The proportion of males, smokers, higher educated and physically active subjects increased across increasing levels of alcohol consumption (table 2).

There was no association between total alcohol consumption and risk of incident VTE ( $p$ for trend across categories: 0.7) (Table 3). Regular alcohol consumption $\geq 7 \mathrm{u} /$ week was associated with a HR of 0.98 (95\% CI: $0.61-1.58)$ compared to teetotalers. 
Hazard ratios for venous thromboembolism (VTE) by different types of alcoholic beverages are shown in table 4. Beer intake was not associated with risk of VTE ( $\mathrm{p}$ for trend 0.5 ). Multivariable HR for $\geq 3 \mathrm{u} /$ week versus teetotalers was 0.97 (95\% CI: 0.62-1.57) (model 2, table 4). However, when adjusted for wine and liquor intake, a beer consumption of $\geq 3$ $\mathrm{u} /$ week tended to be negatively associated with VTE (HR: 0.65, 95\% CI: 0.36-1.19) (model 3). A wine consumption of $\geq 3 \mathrm{u} /$ week was associated with a $22 \%$ reduced risk of VTE (HR: $0.78,95 \%$ CI: $0.47-1.30$ ), though the result was not statistically significant. Further adjustment for beer and liquor intake strengthened the inverse association, and revealed a 47\% reduced risk of VTE (HR: 0.53, 95\% CI: 0.30-1.00) (model 3, table 4). Contrary, liquor consumption was significantly associated with increased risk of VTE ( $p$ for trend 0.04). Subjects with a liquor intake of more than three units per week had a 53\% (Multivariable HR: 1.53, 95\% CI: 1.00-2.33), increased risk of VTE compared to teetotalers after adjustment for age, sex, BMI, smoking, diabetes, cancer, history of CVD, education level and physical activity (model 2, table 4). Further adjustments for wine and beer intake (model 3), revealed an even stronger effect of liquor on VTE (HR 2.03, 95\% CI: 1.15-3.59).

Table 5 shows the association between frequency of binge drinking and VTE among subjects aged $<70$ years. Binge drinking was associated with increased risk of VTE ( $p$ for trend across categories: 0.07). Subjects who reported a binge drinking frequency of once a week or more had an age- and sex-adjusted 1.2-fold (HR: 1.20, 95\% CI: 0.70-2.09) increased risk of VTE compared to teetotalers, and a $63 \%$ increased risk compared to non-binge drinkers who were not teetotalers (HR: 1.63, 95\% CI: 0.96-2.77). Further adjustments for BMI, smoking, diabetes, cancer, history of CVD, education level and physical activity attenuated the risk 
estimates (HR: 1.17, $95 \%$ CI: 0.66-2.09, and HR: 1.47, 95\% CI: 0.85-2.54, respectively) (table 5).

In order to investigate whether liquor or wine consumption was related to specific drinking patterns among subjects in our study, we conducted a cross tabulation between beverage categories and binge drinking. Among those in the upper category of liquor ( $\geq 3 \mathrm{u} /$ week) $36 \%$ reported a binge drinking frequency of once a week or more. The corresponding numbers for wine drinkers was $25 \%$ and $29 \%$ for beer drinkers $(\geq 3 \mathrm{u} /$ week $)$

\section{Discussion}

In the present study, liquor consumption and binge drinking was associated with increased risk of VTE. Subjects who drank three or more units of liquor per week had a 53\% higher risk of VTE compared to teetotalers. Binge drinking at a frequency of once per week or more was associated with a $47 \%$ increased risk of VTE compared to non-binge drinkers who were not teetotalers. In contrast, wine consumption of three or more units per week was associated with a $22 \%$ reduced risk of VTE. The risk estimates associated with liquor intake and wine intake were even stronger after adjustment for the other types of alcoholic beverages; liquor drinkers $(\geq 3 \mathrm{u} /$ week) had $103 \%$ increased risk of VTE, whereas wine drinkers $(\geq 3 \mathrm{u} /$ week) had $47 \%$ reduced risk compared to teetotalers. The latter findings suggest that the observed harmful effect of liquor was not caused by absence of wine intake, and beneficial effect of wine was not caused by absence of liquor intake. Beer consumption showed a possible negative association with VTE only after adjustments for the other types of alcoholic beverages. 
So far, only The Iowa Women's Health Study (14) has reported on the impact of different types of alcoholic beverages on VTE risk. In contrast to our findings, they reported that the observed protective effect of alcohol was significant for beer consumption only, and not for wine or liquor. The discrepancy between these studies may to some extent be explained by differences in cut off values for alcoholic beverages ( $\geq 1 \mathrm{u} /$ week vs. $\geq 3 \mathrm{u} /$ weeks) and study populations (elderly women vs. general population of both genders) as drinking patterns may differ both among genders and age groups.

Previous studies have shown somewhat diverging results on the relation between alcohol and risk of VTE. A prospective cohort of elderly from three different communities in the US (13), found that light to moderate alcohol consumption was associated with a decreased risk of VTE. The MISS study (15), a large Swedish cohort of middle-aged women, found that women with moderate alcohol consumption had lower risk of VTE. In contrast, the LITEstudy, a prospective cohort including middle-aged and elderly subjects from a general population (17), found no association between alcohol consumption and VTE. Moreover, a prospective study, including male physicians only (4), found that alcohol was protective against coronary heart disease, but not associated with stroke or VTE. In a large case-control study of Dutch men and women (16), subjects with a previous history of VTE had lower alcohol intake, and a concomitant decrease in fibrinogen levels in alcohol consumers compared to abstainers was suggested as a plausible explanation for the decreased risk of VTE. In contrast, another case-control study conducted in France, found no association between alcohol and VTE (18). In our study, total alcohol intake showed no association with VTE. However, this was probably explained by the fact that risk estimates for wine and liquor consumption pointed in opposite directions. 
Previous studies investigating the impact of alcohol types on arterial cardiovascular diseases (CVD) have proposed a superior antithrombotic effect of wine compared to beer and liquor $(20,21)$. Accordingly, wine was inversely related to VTE in our study, whereas liquor was associated with a considerable increase in risk of VTE. The differential impact of alcoholic beverages on risk of VTE may suggest that other components of wine, rather than ethanol itself, may be responsible for the beneficial effect. Resveratrol, a polyphenol found in the skin of grapes, promotes antithrombotic effects (22) such as suppression of aberrant expression of tissue factor $(11,22)$, suppression of platelet aggregation (7) and induction of platelet disaggregation in preformed thrombi (23). A possible mechanism for the apparent disadvantage of liquor consumption may be related to the strong association between heavy liquor consumption and the tendency to binge drinking. Binge drinking is known to contradict the beneficial effect of moderate alcohol consumption on the risk of CVD $(12,24)$ and to exert an unfavorable effect on the haemostatic system by inhibiting the fibrinolytic system $(10,25)$. Moreover, a possible wine preference in non-binge drinkers may explain the observed risk differences between frequent binge drinkers, non-binge drinkers and teetotalers (table 5).

Several studies have addressed the issue that the association between moderate alcohol consumption in general, and wine in particular, and the decreased risk of CVD may be due to a selection of subjects sharing this moderate drinking pattern and wine preference $(26,27)$. Moderate wine drinkers generally have higher education (26), are more physically active and have a lower BMI, a better diet and smoke less frequently (27), compared to the non-drinkers, heavy-drinkers and those who choose beer or liquor over wine. Norwegian national statistics show that people with beer and wine preference have higher educational level and income, 
while the opposite trend exist for liquor drinkers (28). To prevent confounding by other lifestyle factors we adjusted for age, sex, BMI, diabetes, cancer, previous CVD, smoking, physical activity and educational level. The tendency of a decreased risk associated with wine, and the increased risk associated with liquor and binge drinking remained after the adjustments. Further adjustments for estrogen use (women only) did not affect our findings.

The prospective design, large number of participants recruited from a general population with high attendance rate, long-term follow-up, and validated VTE-events are the major strengths of this study. All hospital care and radiological imaging in the region is exclusively provided by a single hospital, which enhances the completeness of the VTE registry. The study also has some limitations. Several risk factors are modifiable, and alcohol consumption habits may change over time. This type of non-differential misclassification, generally leads to underestimation of the true association. However, according to Statistics Norway, the consumption of wine increased significantly in the Norwegian population during the followup period, while beer and liquor consumption was more or less unchanged (29). Information of alcohol consumption habits was collected through self-administered questionnaires, and the reliability and validity of this type of assessment can be questioned. However, self-reported intake of alcohol was associated with expected and previously reported beneficial frequency of CVD $(11,12)$ and level of education (30). A previous validation study (31) showed that assessment of alcohol intake from a food frequency questionnaire (FFQ) was suitable for studying diet-disease associations. Unfortunately we did not have information on former drinkers. The alcohol consumption was fairly low in our study population compared to some of the previous studies from other countries. This is in agreement with the WHO Global Status Report on Alcohol which reported that the intake of pure alcohol in Norway was about half of that in the Netherlands, Italy and the US, and only a third of that in France, during the 
period 1993-97 (6). The low alcohol consumption limited our possibilities to give a finer classification of amount of alcohol and explore the impact of heavy drinking on VTE. However, in spite of generally low overall alcohol consumption in our population cohort, we detected a distinct impact of alcohol type and drinking pattern (binge drinking) on the risk of VTE.

In conclusion, our findings suggest a harmful effect of liquor ( $\geq 3$ units/week) and binge drinking, and a beneficial effect of wine and possibly beer consumption on the risk of VTE. Further studies are needed to establish whether these findings are due to constituents other than ethanol in wine and beer, if it is due to ethanol itself in liquor, the unfavorable binge drinking pattern associated with heavy liquor intake, or other unrecognized health-related confounders associated with types of alcoholic beverages.

\section{Disclosures}

The authors reported no potential conflicts of interest. 


\section{References}


Table 1. Characteristics of VTE-events at the time of VTE diagnosis. The Tromso Study, 1994-2007.

\begin{tabular}{lc}
\hline & \% (n) \\
\hline Age (years) & $67.5 \pm 14$ \\
Sex (males) & $47.4(218)$ \\
& \\
Deep vein thrombosis & $64.1(295)$ \\
Pulmonary embolism & $35.9(165)$ \\
Unprovoked VTE & \\
& $41.3(190)$ \\
Clinical risk factors: & \\
Estrogens & \\
Pregnancy/Puerperium & $14.4(35)$ \\
Heredity & $1.2(3)$ \\
Other medical conditions & \\
& $2.8(13)$ \\
Provoking factors: & $21.3(98)$ \\
Surgery & \\
Trauma & \\
Acute medical conditions & $17.4(80)$ \\
Cancer & $6.7(31)$ \\
Immobilization & \\
Other & $15.2(70)$ \\
\hline
\end{tabular}

No provoking factor present at the time of diagnosis

Estrogen $=$ hormone replacement therapy or oral contraceptives

${ }^{\S}$ Heredity $=$ VTE in first degree relative before aged 60 years

"Other diseases within the previous year (e.g. myocardial infarction, ischemic stroke, hearth failure and chronic obstructive pulmonary disease).

${ }^{\#}$ Immobilization $=$ Bed rest $>3$ days, wheelchair, plaster cast, air/automobile travel $>4 \mathrm{~h}<14$ days prior to event.

${ }^{ \pm}$Other specific provoking factors described by a physician in the medical record (e.g. intravascular catheter). 
Table 2. Baseline characteristics across levels of average alcohol consumption (u/week).

The Tromsø Study, 1994-2007. Values are means (SD) or percentages (numbers).

\begin{tabular}{lccccc}
\hline & & \multicolumn{4}{c}{ Alcohol consumption (u/week) } \\
& Teetotaler & $<\mathbf{1}$ & $\mathbf{1 - 2}$ & $\mathbf{3 - 6}$ & $\geq 7$ \\
\hline $\mathrm{n}$ & 3511 & 7524 & 4678 & 9126 & 1823 \\
Person years & 35637 & 81795 & 51564 & 100044 & 19690 \\
Age (years) & $58 \pm 17$ & $49 \pm 16$ & $44 \pm 13$ & $43 \pm 12$ & $44 \pm 12$ \\
Sex (\% males) & $32.5(1140)$ & $35.9(2703)$ & $42.0(1967)$ & $58.8(5362)$ & $82.1(1496)$ \\
BMI (kg/m²) & $26.2 \pm 4.6$ & $25.3 \pm 4.2$ & $24.8 \pm 3.5$ & $24.8 \pm 3.4$ & $25.4 \pm 3.5$ \\
Smoking (\%) & $20.8(731)$ & $34.9(2627)$ & $33.8(1582)$ & $40.9(3728)$ & $51.0(930)$ \\
Diabetes (\%) & $4.9(172)$ & $1.8(138)$ & $1.5(68)$ & $0.9(85)$ & $0.8(15)$ \\
Cancer (\%) & $5.8(203)$ & $3.5(267)$ & $2.0(94)$ & $1.9(175)$ & $1.5(28)$ \\
CVD (\%) & $15.4(542)$ & $7.8(590)$ & $4.2(196)$ & $3.8(344)$ & $5.0(91)$ \\
Education (\%) & $14.2(499)$ & $21.0(1581)$ & $34.5(1612)$ & $37.9(3459)$ & $41.1(749)$ \\
Physical activity (\%) & $17.1(601)$ & $23.7(1783)$ & $32.1(1503)$ & $38.2(3485)$ & $36.9(672)$ \\
Hormone therapy (\%) & $6.9(163)$ & $11.3(546)$ & $13.9(377)$ & $16.4(616)$ & $17.4(57)$ \\
\hline
\end{tabular}

*Self-reported history of myocardial infarction, angina pectoris or stroke

${ }^{\dagger}$ Education at university/college level

\#Hormone therapy: Self-reported current use of estrogen supplementation or oral contraceptives. Percentage of women. 
Table 3. Hazard ratios (HR) with $95 \%$ confidence interval (CI) for venous

thromboembolism (VTE) by categories of total alcohol intake (units/week). The Tromsø Study, 1994-2007.

\begin{tabular}{|c|c|c|c|c|}
\hline & $\begin{array}{c}\text { Person- } \\
\text { years } \\
\end{array}$ & $\begin{array}{l}\text { VTE- } \\
\text { events }\end{array}$ & $\begin{array}{c}\text { Model 1* } \\
\text { HR (95\% CI) }\end{array}$ & $\begin{array}{c}\text { Model 2 } \\
\text { HR }(95 \% \text { CI) } \\
\end{array}$ \\
\hline \multicolumn{5}{|c|}{ Total alcohol (u/week) } \\
\hline Teetotaler & 35637 & 108 & 1.00 (reference) & 1.00 (reference) \\
\hline$<1$ & 81795 & 135 & $0.85(0.66-1.10)$ & $0.89(0.69-1.16)$ \\
\hline $1-2$ & 76051 & 107 & $1.00(0.75-1.33)$ & $1.10(0.82-1.38)$ \\
\hline $3-6$ & 59851 & 69 & $0.93(0.67-1.29)$ & $0.98(0.70-1.39)$ \\
\hline$\geq 7$ & 19690 & 25 & $0.94(0.59-1.49)$ & $0.98(0.61-1.58)$ \\
\hline$p$ for trend & & & 1.0 & 0.7 \\
\hline
\end{tabular}

*Model 1: Adjusted for age and sex

${ }^{\dagger}$ Model 2: Adjusted for age, sex, body mass index, smoking, diabetes, cancer, history of CVD, education level and physical activity. 
Table 4. Hazard ratios (HR) with $95 \%$ confidence interval (CI) for venous

thromboembolism (VTE) by different types of alcoholic beverages. The Tromsø Study, 1994-2007.

\begin{tabular}{|c|c|c|c|c|c|}
\hline & $\begin{array}{c}\text { Person- } \\
\text { years }\end{array}$ & $\begin{array}{l}\text { VTE- } \\
\text { events }\end{array}$ & $\begin{array}{c}\text { Model 1* } \\
\text { HR (95\% CI) }\end{array}$ & $\begin{array}{c}\text { Model 2 } \\
\text { HR }(95 \% \text { CI) } \\
\end{array}$ & $\begin{array}{c}\text { Model 3 } \\
\text { HR }(95 \% \text { CI) } \\
\end{array}$ \\
\hline \multicolumn{6}{|l|}{ Beer (u/week) } \\
\hline Teetotaler & 35637 & 108 & 1.00 (reference) & 1.00 (reference) & 1.00 (reference) \\
\hline$<1$ & 158750 & 263 & $0.92(0.72-1.16)$ & $0.97(0.76-1.23)$ & $0.73(0.50-1.07)$ \\
\hline $1-2$ & 67905 & 61 & $0.78(0.55-1.10)$ & $0.82(0.58-1.18)$ & $0.59(0.36-0.97)$ \\
\hline$\geq 3$ & 26438 & 28 & $0.98(0.62-1.54)$ & $0.97(0.62-1.57)$ & $0.65(0.36-1.19)$ \\
\hline$p$ for trend & & & 0.4 & 0.5 & 0.7 \\
\hline \multicolumn{6}{|l|}{ Wine (u/week) } \\
\hline Teetotaler & 35637 & 108 & 1.00 (reference) & 1.00 (reference) & 1.00 (reference) \\
\hline$<1$ & 168911 & 253 & $0.91(0.72-1.16)$ & $0.95(0.75-1.22)$ & $0.73(0.51-1.06)$ \\
\hline $1-2$ & 65173 & 79 & $0.92(0.68-1.25)$ & $0.99(0.72-1.36)$ & $0.73(0.46-1.14)$ \\
\hline$\geq 3$ & 19009 & 20 & $0.73(0.45-1.19)$ & $0.78(0.47-1.30)$ & $0.53(0.30-1.00)$ \\
\hline$p$ for trend & & & 0.3 & 0.5 & 0.1 \\
\hline \multicolumn{6}{|l|}{ Liquor (u/week) } \\
\hline Teetotaler & 35637 & 108 & 1.00 (reference) & 1.00 (reference) & 1.00 (reference) \\
\hline$<1$ & 185293 & 229 & $0.83(0.65-1.05)$ & $0.88(0.69-1.13)$ & $1.10(0.75-1.62)$ \\
\hline $1-2$ & 53317 & 90 & $1.11(0.82-1.50)$ & $1.18(0.86-1.61)$ & $1.53(0.96-2.44)$ \\
\hline$\geq 3$ & 14483 & 33 & $1.48(0.98-2.24)$ & $1.53(1.00-2.33)$ & $2.03(1.15-3.59)$ \\
\hline$p$ for trend & & & 0.07 & 0.04 & 0.001 \\
\hline
\end{tabular}

*Model 1: Adjusted for age and sex

†Model 2: Adjusted for age, sex, body mass index, smoking, diabetes, cancer, history of CVD, education level and physical activity.

*Model 3: Categories of beer, wine or liquor added to model 2 making the types of alcoholic beverages adjusted for one another (i.e. beer is adjusted for wine intake, liquor intake age, sex, body mass index, smoking, diabetes, cancer, history of CVD, education level and physical activity). 
Table 5. Hazard ratios (HR) with 95\% confidence interval (CI) for venous thromboembolism (VTE) by frequency of binge drinking in subjects $<70$ years old $(n=21$ 382). The Tromsø Study, 1994-2007.

\begin{tabular}{|c|c|c|c|c|c|c|}
\hline $\begin{array}{l}\text { Frequency of binge } \\
\text { drinking }\end{array}$ & $\begin{array}{l}\text { Person- } \\
\text { years }\end{array}$ & $\begin{array}{l}\text { VTE- } \\
\text { events }\end{array}$ & $\begin{array}{c}\text { Model 1* } \\
\text { HR (95 \% CI) }\end{array}$ & $\begin{array}{c}\text { Model 2 } \\
\text { HR (95 \% CI) }\end{array}$ & $\begin{array}{c}\text { Model 1* } \\
\text { HR (95 \% CI) }\end{array}$ & $\begin{array}{c}\text { Model 2 }^{\dagger} \\
\text { HR (95 \% CI) }\end{array}$ \\
\hline Teetotaler & 25833 & 50 & 1.00 (reference) & 1.00 (reference) & - & - \\
\hline Never & 62722 & 68 & $0.74(0.51-1.07)$ & $0.81(0.56-1.18)$ & 1.00 (reference) & 1.00 (reference) \\
\hline A few times per year & 91220 & 98 & $0.97(0.67-1.39)$ & $1.03(0.72-1.50)$ & $1.32(0.96-1.82)$ & $1.30(0.94-1.80)$ \\
\hline $1-2$ times per month & 43703 & 45 & $1.10(0.71-1.70)$ & $1.11(0.71-1.76)$ & $1.15(1.01-2.25)$ & $1.42(0.94-2.14)$ \\
\hline$\geq$ Once a week & 13386 & 19 & $1.20(0.70-2.09)$ & $1.17(0.66-2.09)$ & $1.63(0.96-2.77)$ & $1.47(0.85-2.54)$ \\
\hline$P$ for trend & & & 0.07 & 0.3 & 0.02 & 0.2 \\
\hline
\end{tabular}

*Model 1: Adjusted for age and sex

${ }^{\dagger}$ Model 2: Adjusted for age, sex, body mass index, smoking, diabetes, cancer, history of CVD, education level and physical activity. 


\section{What is known about this topic}

- Previous studies on the relation between alcohol consumption and VTE have shown diverging results.

- The association between types of alcoholic beverages and venous thromboembolism (VTE) is not well described.

\section{What this paper adds}

- There was no association between total alcohol consumption and VTE. However, the risk estimates for VTE by different types of alcoholic beverages pointed in opposite directions.

- Liquor consumption and binge drinking was associated with increased risk of VTE, respectively, whereas wine consumption was associated with reduced risk of VTE. 